\title{
RF Based Pick and Place Robot
}

\author{
M. Kalpana Chowdary, M. Sirisha, T. Vishnu Priya, N.Charanya \\ ${ }^{I}$ (Assistant Professor, Department of Electronics and Communication Engineering, Chalapathi Institute of \\ Engineering and Technology, Andhra Pradesh, India, \\ ${ }^{2}$ (Assistant Professor, Department of Electronics and Communication Engineering, Chalapathi Institute of \\ Engineering and Technology, Andhra Pradesh, India, \\ ${ }^{3}$ (Student, Department of Electronics and Communication Engineering, Chalapathi Institute of Engineering and \\ Technology, Andhra Pradesh, India, \\ ${ }^{4}$ (Student, Department of Electronics and Communication Engineering, Chalapathi Institute of Engineering and \\ Technology, Andhra Pradesh, India,
}

\begin{abstract}
Pick $n$ place robot is very interesting, although this type of concept based circuits developed almost 10 years back ago. In previous pick $n$ place robot circuits they may use Bluetooth or infrared, but in this we use radio frequency. Here in this, we use keypad which enables switching "forward, backward, right and left" to pick \& place an object. It can be used to move the robot from any distance over limited range.

Index terms: Bluetooth, Infrared, Radio frequency
\end{abstract}

\section{Introduction}

Robotics is the branch of engineering science \& Technology related to robots, and their design, manufacture, application, and structural disposition. Robotics is related to electronics, mechanics, and software. Robotics research today is focused on developing systems that exhibit modularity, flexibility, redundancy, faulttolerance, a general and extensible software environment and seamless connectivity to other machines, some researchers focus on completely automating a manufacturing process or a task, by providing sensor based intelligence to the robot arm, while others try to solidify the analytical foundations on which many of the basic concepts in robotics are built.

In this highly developing society, time and man power are critical constraints for completion of task in large scales. The automation is playing important role to save human efforts in most of the regular and frequently carried works. One of the major and most commonly performed works is picking and placing of objects from source to destination. A pick and place robot is the one which is used to pick up an object and place it in the desired location. It can be a cylindrical robot providing movement in horizontal, vertical and rotational axes.

\section{Technology}

This radio frequency $(\mathrm{RF})$ transmission system employs amplitude shift keying (ASK) with transmitter operating at $434 \mathrm{MHz}$. The address and control bits are received by the Encoder IC(HT12E) in the form of parallel data. The control signals from remote switches with 8 address bits constitute a set of 12 parallel signals. The encoder retrieves the original data from the received serial data and then sends to the microcontroller. The encoder HT12E encodes the parallel signals into serial bits from encoder IC HT12E transmits to HT12D. The decoder then retrieves the original parallel format from the received serial data.

\section{System Description}

In this system, a pick and place robot is controlled with the push-buttons arranged in the remote. On pressing a corresponding button, a command signal is transmitted to the robot such that the robot moves in the appropriate direction. This project consists of two blocks: transmitter and receiver block. The commands send by a user from the transmitter block are received by the receiver block to perform desired actions. Whenever we press any key, particular data line is grounded. When TE pin is low (TE-transmission enable pin is low active) the address and data are transmitted serially through Data Out pin. When $5 \mathrm{~V}$ supply is given to circuit all the data lines are low and because of invertors all four outputs of the inverters will be high. Whenever you press any switch from transmitter, then address \& data are transmitted together, then RF receiver will receive the data and gives this address \& data to IC HT12D. 


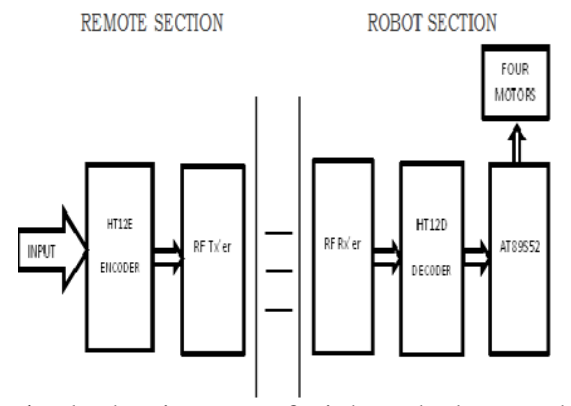

Fig.1 Block Diagram of Pick and Place Robot

IC HT12D first compares the address three times and if it matches, it gives high pulse on VT pin and latch the data.HT12D receives the signals compares address thrice, gives high pulse on pin VT and then latches the data. Simply you can see which ever switch is pressed on TX side that particular data line is high on Rx.Now by connecting the data lines to motor driver IC we can able to control the motors. [1]

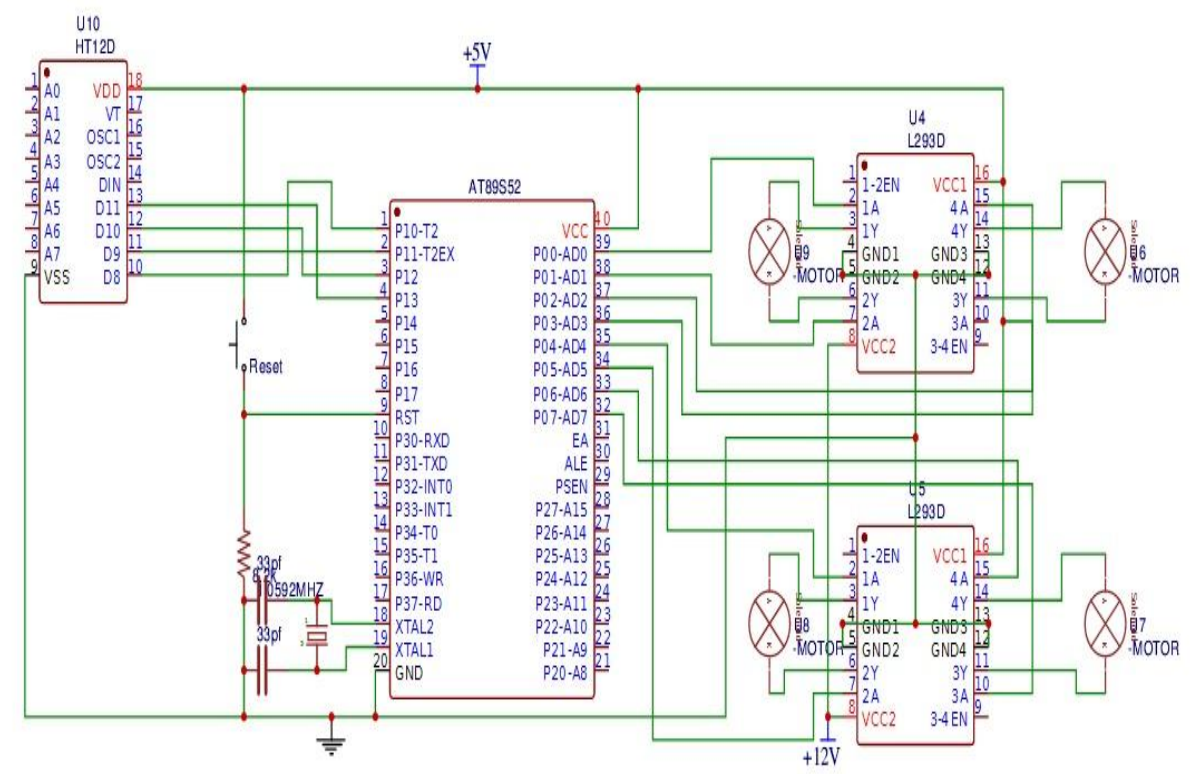

Fig.2 Pin description of pick and place robot

The transmitter part consists of the keypad interfaced to the microcontroller. Any button number in decimal format is converted to 4 digit binary by the microcontroller and the parallel output at one of its port is applied to the encoder. The encoder converts this parallel data to serial data and this is fed to thetransmitter, fitted with an antenna to transmit the serial data. The receiver side consists of a decoder interfaced to the microcontroller. The decoder converts the received command in serial format to the parallel form and gives this data to the microcontroller.

\subsection{Microcontroller}

The AT89S52 is a low-power, high-performance CMOS 8-bit microcomputer with 8K bytes of Flash programmable and erasable read only memory. The device is manufactured using Atmel's highdensity nonvolatile memory technology and is compatible with the industry-standard 8051 instruction set and pin out. The on-chip Flash allows the program memory to be reprogrammed in-system or by a conventional nonvolatile memory programmer. By combining a versatile 8-bit CPU with Flash on a monolithic chip, the Atmel AT89C51 is a powerful microcomputer, which provides a highly flexible and cost-effective solution to many embedded control applications.

\section{Features}

8051 architecture consists of these features:

1. Eight bit CPU with registers A and B

2. Sixteen bit program counter and data pointer 
3. Eight bit program status word

4. Eight bit stack pointer

5. Internal ROM of $4 \mathrm{~K}$

6. Internal RAM of 128 bytes are

a) Four register banks, each containing eight registers

b) Sixteen bytes, which may be addressed at bit level

c) Eighty bytes of general-purpose data memory

7. 32 Programmable I/O Lines

8. Two 16-bit Timer/Counters

9. Full Duplex UART Serial Channel

10. Control register: TCON, TMOD, SCON, PCON, and IP. [2]

\subsection{RF Module}

The RF module, as the name suggests, operates at Radio Frequency. The corresponding frequency range varies between $30 \mathrm{kHz} \& 300 \mathrm{GHz}$. In this $\mathrm{RF}$ system, the digital data is represented as variations in the amplitude of carrier wave. This kind of modulation is known as Amplitude Shift Keying (ASK).The RF module comprises of an RF Transmitter and an RF Receiver. The transmitter/receiver (Tx/Rx) pair operates at a frequency of $433 \mathrm{MHz}$ and it has 4 output pins i.e. it can operate 4 peripherals remotely. The RF module is often used along with a pair of encoder/decoder. The encoder is used for encoding parallel data for transmission feed while reception is decoded by a decoder.HT12D-HT12E,HT640-HT648, etc. are some commonly used encoder/decoder pair ICs.

\subsection{RF Transmitter}

An RF transmitter module is a small PCB sub-assembly capable of transmitting a radio wave and modulating that wave to carry data.HT12E Encoder IC will convert the 4 bit parallel data given to pins D0 - D3 to serial data. This output serial data is given to ASK RF Transmitter. Address inputs A0 - A7 can be used to provide data security and can be connected to GND (Logic ZERO) or left open (Logic ONE). Status of these Address pins should match with status of address pins in the receiver for the transmission of the data [4]. Data will be transmitted only when the Transmit Enable pin (TE) is LOW.

\subsection{RF Receiver}

An RF receiver module receives the signal, and demodulates it. ASK RF Receiver receives the data transmitted using ASK RF Transmitter. HT12D decoder will convert the received serial data to 4 bit parallel data D0 - D3. The status of these address pins A0-A7 should match with status of address pin in the HT12E at the transmitter for the transmission of data [5].

\subsection{Drivers}

L293D is a typical Motor driver IC which allows DC motor to drive on either direction. L293D is a 16-pin IC which can control a set of two DC motors simultaneously in any direction. It means that you can control two DC motor with a single L293D IC. So to drive the motors we need drivers [6].

\subsection{Motors}

Here we use four DC motors. Two DC motors for moving purpose and other two for pick and place purpose.

Table 1: Motor Directions

\begin{tabular}{|l|l|l|l|l|}
\hline S.NO & Motor-1 & Motor-2 & Motor-3 & Motor-4 \\
\hline Forward & clock & clock & none & none \\
\hline Backward & Anti clock & Anti clock & none & none \\
\hline Left & stop & clock & none & none \\
\hline Right & clock & stop & none & none \\
\hline Pick & none & none & clock & clock \\
\hline Place & none & none & $\begin{array}{l}\text { Anti } \\
\text { clock }\end{array}$ & Anti clock \\
\hline
\end{tabular}

If motor-1 and motor- 2 are in clockwise direction robot move in forward direction. If motor-1 and motor- 2 are in anti-clockwise direction robot move in backward direction. If motor- 1 is stop and motor- 2 is in clockwise direction robot move in left direction. If motor-2 is stop and motor-1 is in clockwise direction 
robot move in right direction. If motor-3 and motor-4 are in clockwise direction it can pick an object. If motor-3 and motor-4 are in anti-clockwise direction it can place an object [3].

\section{Experimental Results}

It picks an object from one location and place an object to the desired location.

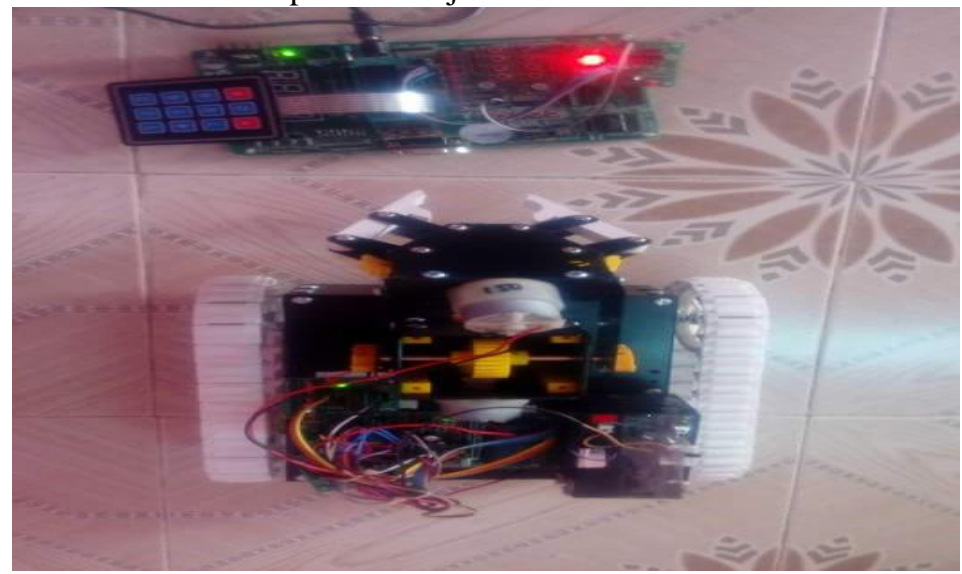

Fig.3 Physical Structure of Robot

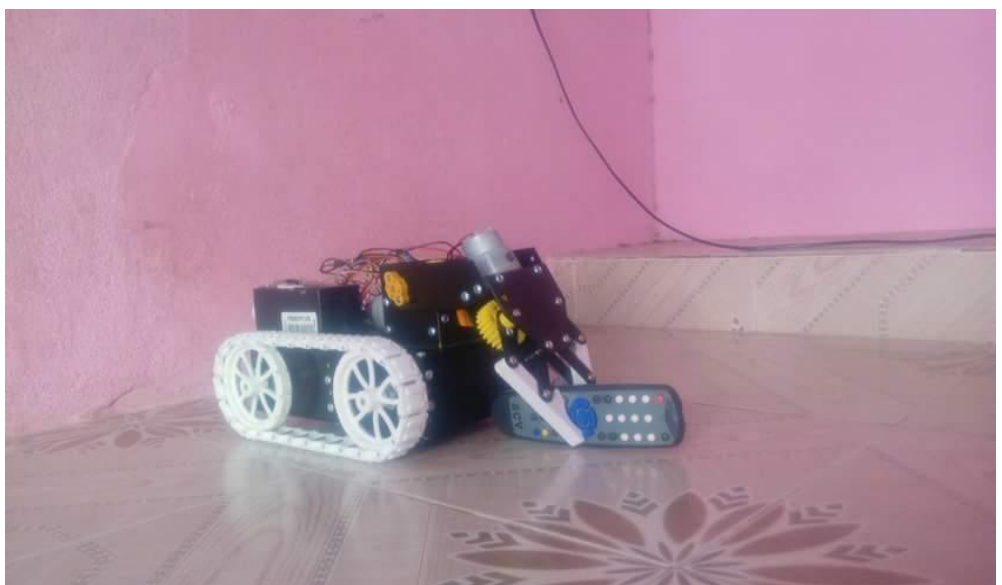

Fig.4 Pick Condition

The above figure shows the Robot is in the object pick condition, which holds an object by using arm and moves to destination.

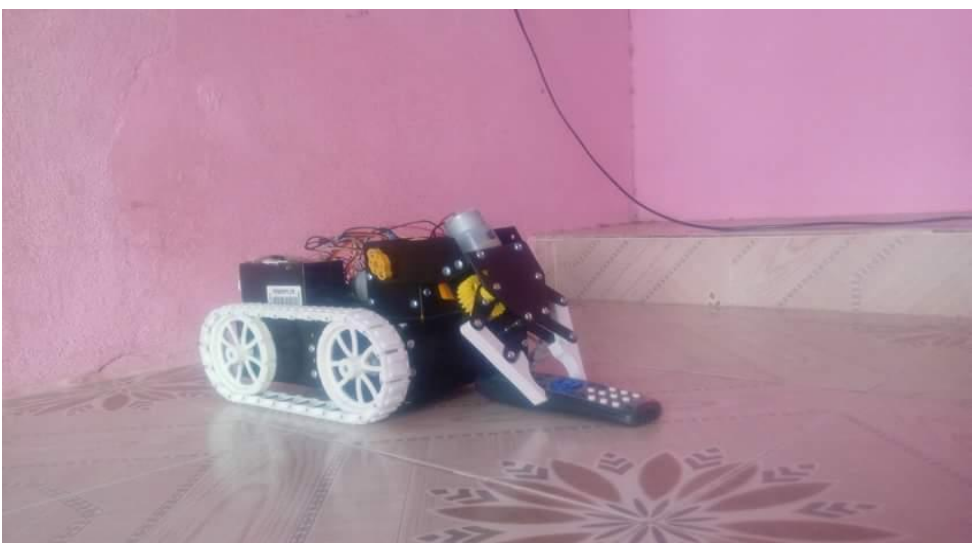

Fig.5 Placing Position

The above figure 5 shows the object placing condition. 


\section{Advantages And Applications}

- They are faster and can get the work done in seconds compared to their human counterparts.

- They are flexible and have the appropriate design.

- They are accurate.

- They increase the safety of the working environment and actually never get tired.

\section{Medical applications}

These robots can be used in various surgical operations like in joint replacement operations. They perform the operations with more precision and accuracy.

\section{Industrial applications}

These robots are used in manufacturing segments to pick up the required parts and place them in correct positions to complete the machinery fixture.

\section{Defense applications}

They can be used for robot performed military applications such as surveillances and also to pick up harmful objects like bombs and diffuse them safely.

\section{Conclusion}

In this prototype project, we design in such away that this robot can be moved anywhere and it can place any object in the desired location. It is very much useful in the places where a human cannot go to into the places like ground canals, smoke oriented caves. Hence this project is very much useful in this situations.

\section{References}

[1]. Robotic Enginnering by Richard D Klafter, Thomas A chmielewski, Michael Negin

[2]. Atmel AVR Microcontroller Primer: Programming and Interfacing By: Steven F. Barret, Daniel J. Pack

[3]. RatheeshRajan "Foundation Studies for an Alternate Approach to Motion Planning of Dynamic Systems" M.S.E., the University of Texas at Austin, 2001

[4]. RK Mittal and IJ Nagarath "Robotics and Control” BITS Pilani, 2003

[5]. Kuan Pei Wen, "PICK AND PLACE ROBOTIC ARM", Under Graduate Project Paper Submitted to Universiti Teknologi Malaysia. June 2012

[6]. Mohamed Naufal, Omar. "Pick And Place Robotic Arm Controlled By Computer." (2007). 